Cipango Cahiers d'études japonaises

Hors-série | 2008

Autour du Genji monogatari

\title{
Temps et espace dans le Roman de la Chambre basse
}

Time and space in the Tale of the Sunken Room

\section{Daniel Struve}

\section{(2) OpenEdition}

Journals

Édition électronique

URL : https://journals.openedition.org/cipango/594

DOI : $10.4000 /$ cipango.594

ISSN : 2260-7706

Éditeur

INALCO

Édition imprimée

Date de publication : 1 janvier 2008

Pagination : 89-108

ISBN : 978-2-85837-170-5

ISSN : $1164-5857$

Référence électronique

Daniel Struve, "TEMPS ET ESPACE DANS LE ROMAN de LA CHAMBre BASSE », Cipango [En ligne], Horssérie | 2008, mis en ligne le 11 juin 2012, consulté le 30 juin 2021. URL : http://

journals.openedition.org/cipango/594; DOI : https://doi.org/10.4000/cipango.594

Ce document a été généré automatiquement le 30 juin 2021.

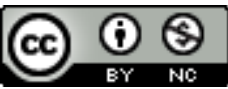

Cipango est mis à disposition selon les termes de la Licence Creative Commons Attribution - Pas d'Utilisation Commerciale 4.0 International. 


\title{
Temps et espace dans le Roman de la Chambre basse
}

Time and Space in the Tale of the Sunken Room

\author{
Daniel Struve
}

1 L'importance de la place prise par le Roman du Genji dans l'histoire de la littérature japonaise a sans doute contribué à l'oubli dans lequel sont tombées nombre d'œuvres antérieures ou contemporaines. Beaucoup de romans ont été perdus ${ }^{1}$. Parmi les rares dont le texte a été transmis à la postérité, aucun ne peut rivaliser avec le chef-d'œuvre du genre. Si le Taketori monogatari (Le Conte du Coupeur de bambou), qualifié d'ancêtre du genre par le Roman du Genji, est resté célèbre, l’Utsuho monogatari うつほ物語 (Roman de la Caverne ${ }^{2}$ ) et l'Ochikubo monagatari 落洼物語 (Roman de la Chambre basse ${ }^{3}$ ) sont peu lus et relativement peu étudiés. Pourtant, ces œuvres sont chacune à sa manière remarquables et méritent de retenir l'attention par leurs qualités propres. Elles présentent en outre l'intérêt de nous renseigner sur la situation du genre romanesque au Japon à la fin du $\mathrm{X}^{\mathrm{e}}$ siècle et de mieux faire comprendre par comparaison l'originalité du Roman du Genji, en même temps que ce qu'il doit aux romans antérieurs dont il constitue une sorte de somme critique.

2 Le Roman de la Chambre basse a pu être considéré comme une œuvre secondaire, fruit d'une période où le genre n'avait pas encore atteint sa maturité, une œuvre de fiction populaire, destinée à des lecteurs peu érudits et faite pour plaire aux femmes de noblesse intermédiaire au service des grandes familles ${ }^{4}$. Cette caractéristique, qui a le défaut de transposer dans une époque très différente de la nôtre des catégories comme celle de «littérature populaire » qui n'y ont guère de sens, nous semble manquer complètement la véritable nature de ce roman de mœurs, dont l'inspiration comique est prise à tort pour une marque d'infériorité. Nous pensons au contraire que le Roman de la Chambre basse, qui était tenu en grande estime par Ueda Akinari au XVIII siècle ${ }^{5}$, est au contraire le témoin d'une étape où le roman est déjà très élaboré et conscient de ses moyens. S'il s'agit, à l'évidence, d'une œuvre beaucoup plus modeste par ses dimensions et par son propos que le Roman du Genji, cela ne diminue en rien sa valeur ni son intérêt historique et littéraire ${ }^{6}$. 
3 Le Roman de la Chambre basse est l'histoire des persécutions que subit de la part d'une marâtre autoritaire et acariâtre une jeune fille d'ascendance impériale. Mal protégée par le conseiller moyen, son père, celle-ci se voit assigner au sein de la famille un rang inférieur à celui des enfants de la première épouse. Touché par le sort malheureux de la jeune princesse, Michiyori, le fils du ministre de droite, lui-même promis à un brillant avenir, lui rend visite, puis, tombé amoureux, en fait son épouse, l'enlève et s'attache à la venger et à punir méthodiquement ses persécuteurs. La fin du roman évoque la réconciliation, une fois la vengeance accomplie, des deux familles. Le thème, sans doute emprunté au folklore, de la belle-fille maltraitée devait trouver des échos dans la société polygame de l'époque de Heian? ${ }^{7}$. En tout état de cause, il est ici traité sur le mode du roman et non du conte populaire. Ainsi, l'action est située dans un temps proche de celui des premiers lecteurs, et dans l'espace familier des demeures aristocratiques de la capitale. La situation politique décrite est celle qui prévalait à la fin du $\mathrm{X}^{\mathrm{e}}$ siècle, où une branche de la famille des Fujiwara, après avoir écarté ses rivaux, obtient le monopole du pouvoir et le contrôle des richesses du pays par l'intermédiaire des gouverneurs de province, enrôlés dans sa clientèle. Comme l'ont relevé les critiques, le roman se caractérise également par l'absence de tout élément de merveilleux ou de surnaturel. Certes, le sort uniformément heureux réservé aux personnages positifs, par opposition à la rétribution qui attend les méchants, élimine tout élément de hasard dans le déroulement d'ensemble de l'intrigue et montre que la rupture avec l'univers du conte n'est pas complète. Cependant, c'est par le seul jeu des actions humaines que le plan du destin se réalise : le triomphe politique du vertueux et fidèle Michiyori et de sa famille met fin au désordre et ramène l'harmonie dans la famille troublée du conseiller moyen, promu Grand Conseiller.

4 Dans cette étude, nous bornerons notre analyse à la seule première partie du roman, où sont évoquées les premières années de l'héroïne, la cour que lui fait le fils du ministre de droite, le conflit que cette liaison provoque avec la marâtre, conduisant à l'enfermement de l'héroïne, puis à son enlèvement par Michiyori au début de la seconde partie. Nous voudrions montrer comment l'organisation temporelle et spatiale du roman dans cette première partie crée un univers proprement dramatique, propice au déploiement des conflits entre les personnages et entre les valeurs dont ils sont porteurs.

\section{Structure temporelle de la première partie}

5 Le roman commence par un préambule où est présentée rapidement la situation qui prévaut dans la famille du conseiller moyen, et les circonstances difficiles où se trouve l'héroïne, surnommée par sa marâtre "Demoiselle de la chambre basse». La présentation de l'héroïne, de ses malheurs, de sa beauté et de ses talents de musicienne, s'accompagne de celle de sa fidèle suivante Akoki, issue, comme on l'apprend plus tard, d'une famille de rang gouvernoral. Le fils du ministre de droite, Michiyori, apprend du mari d'Akoki l'existence de la demoiselle et entreprend alors de lui envoyer des lettres qui restent sans réponses, puis profite de circonstances favorables pour lui rendre visite pendant trois nuits consécutives, ce qui scelle le mariage. Cependant, la tension dramatique est relancée par l'augmentation soudaine du travail de couture exigée de la Demoiselle et par la perte d'une correspondance interceptée par la marâtre. Désormais consciente de la présence indésirable d'un époux et sentant son pouvoir menacé, la 
marâtre décide de calomnier la Demoiselle auprès de son père et l'enferme dans un entrepôt proche de ses appartements, établissant un contrôle entier sur sa belle-fille. $\mathrm{Au}$ début de la seconde partie, la Demoiselle subit les assauts d'un prétendant indésirable avant d'être délivrée de cette épreuve par Michiyori.

6 Ainsi, nous avons à faire dans cette première partie à une structure temporelle complexe, dont l'auteur se sert pour relancer à loisir la tension dramatique. Dès le début se met en branle une temporalité familiale : deux des quatre filles du conseiller moyen ont procédé à la cérémonie de la prise du mo (mogi 裳着9) et sont en pouvoir de mari, tandis que les deux dernières ne sont pas encore mariées, comme ne l'est pas non plus l'héroïne principale du roman. La prise de mo et le mariage de la Demoiselle troisième, qui se voit attacher les services d'Akoki, constitue le premier événement du roman. Inséré dans la partie introductrice, il accentue le sentiment d'isolement et d'humiliation de la Demoiselle de la chambre basse, chargée de coudre les vêtements pour le nouveau gendre. Un peu plus tard commenceront les pourparlers en vue de la Demoiselle quatrième, que l'on prévoit de donner justement à Michiyori, ce qui fait d'elle la rivale de la Demoiselle de la chambre basse. Ainsi, le motif du mariage introduit une tension et une dynamique temporelle, soulignant l'isolement de l'héroïne au sein de la famille. La Demoiselle se trouve au centre d'une temporalité propre, distincte de celle de la maison, ce dont elle a une conscience de plus en plus aiguë à mesure que lui apparaît le caractère insupportable de sa condition, au point de souhaiter trouver refuge dans la mort.

$7 \quad$ La fin du prologue et le début de l'action romanesque proprement dite sont marqués par une date: «ce devait être le premier jour du huitième mois ». Peu après cette première indication précise, l'inquiétude du père de l'héroïne devant le manque de vêtement de sa fille mal-aimée, introduit le thème des saisons et crée un premier sentiment d'urgence. Des indications saisonnières sont ensuite contenues dans les lettres de Michiyori : la seconde est attachée à un brin de miscanthe en fleur et joue avec les associations traditionnelles de ce $\operatorname{mot}^{10}$, la suivante fait allusion aux averses qui marquent la fin de l'automne et le début de l'hiver : à la fin du neuvième et au début du dixième mois. Ces deux éléments sont puisés dans le réservoir canonique des mots de saisons de la poésie japonaise, rigoureusement codifiés. Un peu plus tard, les premières visites de Michiyori à la faveur d'un départ de la famille en pèlerinage ont lieu sous une pluie battante, qui permet de situer l'ensemble de l'épisode au dixième mois. Les éléments de la nature font irruption dans les relations entre les personnages, mettant à l'épreuve les sentiments du héros et lui donnant l'occasion de se montrer un amant exemplaire. Une fois passé ce cap, le repérage se fait par rapport à la fête extraordinaire de Kamo située le 27 du onzième mois, au milieu de l'hiver. L'épisode de l'enlèvement au début du second livre intègre habilement le thème saisonnier : c'est un long séjour sur la véranda glacée qui met hors de combat le vieil Apothicaire donné pour mari à la Demoiselle.

8 À ce temps du calendrier et de la nature où sont pris les personnages, se superpose une temporalité sociale qui, dans cette première partie, est pour l'essentiel celle d'une grande maison aristocratique: le mariage des filles, dont nous avons déjà parlé, la préparation des vêtements pour les différentes occasions, qui rejaillit par un surcroît de travail sur la vie de l'héroïne, les fêtes et les pèlerinages. C'est le départ de l'ensemble de la famille à l'exception de la Demoiselle pour un pèlerinage de plusieurs jours au temple d'Ishiyama, qui marque le premier grand tournant du roman. La fête 
extraordinaire du sanctuaire de Kamo rompt le fragile équilibre introduit par le mariage clandestin de Michiyori avec la Demoiselle, en imposant une charge insupportable à celle-ci. Le début des négociations en vue du mariage de la fille cadette avec la famille de Michiyori, sur laquelle la marâtre a jeté son dévolu, contribue à la tension dramatique en introduisant de nouvelles échéances. Cet événement sera pleinement exploité dans la seconde partie. La cour elle-même de Michiyori obéit à des codes sociaux extrêmement précis, qui sont intégrés dans l'élaboration du temps romanesque : une correspondance commencée doit se poursuivre quelque temps avec des interruptions; la première visite, pour se transformer en un véritable mariage, doit être réitérée deux fois, et s'achever à la troisième nuit par la consommation de gâteaux de riz ; une visite doit être suivie par une lettre, etc.

9 Dans ce double cadre naturel (cosmique) et social (familial) s'inscrivent les multiples projets souvent incompatibles des personnages : l'aspiration de l'héroïne à mourir pour échapper à sa condition ; la cour de plus en plus insistante, puis l'amour exclusif que lui voue Michiyori qui vient à bout de ce désespoir comme des obstacles extérieurs; les velléités du général de Katano, figure de Don Juan empruntée à d'autres romans de l'époque, qui stimule la jalousie de Michiyori ${ }^{11}$; la rapacité de la marâtre et ses manigances, motivées en premier lieu par un besoin constant en vêtements habilement cousus pour ses gendres - c'est ce qui la conduit à séquestrer finalement la Demoiselle et en même temps à lui laisser la vie sauve - mais aussi l'appétit de pouvoir et la jalousie. À tous ces éléments, il faut encore ajouter les menées d'Akoki et de son mari, déterminés à venir en aide à leurs maîtres, mais pas toujours d'accord sur la manière de procéder. Akoki, notamment, joue un rôle de tout premier plan dans cette première partie du roman. Ses récriminations envers son mari, ses premières réserves concernant Michiyori dont elle met en doute la constance, les plans qu'elle échafaude constamment jusque dans le moindre détail et son activité débordante pour venir en aide à sa maîtresse lui confèrent une place centrale dans le récit et dans la construction de sa temporalité.

10 L'interaction de toutes ces séries temporelles et causales crée une trame narrative très serrée, qui permet, à certains moments du récit, de suivre les éléments jour après jour et même heure après heure. Si les premiers événements, ponctués par l'envoi répété, mais irrégulier, de lettres laissées sans réponse ne sont situés dans le temps que d'une manière assez vague, le repérage devient ensuite beaucoup plus précis. Ainsi, le départ de la famille à Ishiyama ouvre une durée continue de sept jours (six nuits), remplie par les échanges de messages, les visites de Michiyori, les préparatifs précédant ces visites. Une seconde période commence le 23 du onzième mois avec l'annonce de la désignation du mari de la troisième fille de la maison pour danser à la fête extraordinaire de Kamo. Les événements s'enchaînent dès lors d'une manière continue jusqu'à la fin de la première partie. Ils se prolongent même au-delà, au début de la seconde, jusqu'au matin du 27, jour de la fête, où Michiyori vient enlever la Demoiselle. Certes, la suite des événements est quelque peu brouillée dans la première journée de la séquestration ${ }^{12}$. Cependant, dans l'ensemble, ces deux longs épisodes continus témoignent d'une grande maîtrise dans la construction et la conduite du récit.

11 Un passage, situé au point de contact des deux épisodes, a particulièrement intrigué les commentateurs :

Accepteriez-vous de venir là où je voudrais vous installer?" "Ce sera comme vous voudrez ", répondit la Demoiselle. "Très bien! " fit le capitaine et il continuait à s'entretenir avec elle, sans se lever. 
C'était le vingt-trois du onzième mois. Le capitaine attaché à la Chancellerie privée, mari de la Demoiselle troisième, fut soudainement désigné pour danser à la fête extraordinaire du sanctuaire de Kamo et la Dame épouse s'employa précipitamment aux préparatifs. Akoki se dit avec angoisse que les travaux de couture allaient sûrement retomber sur sa maîtresse et en effet une servante vint apporter la découpe d'un pantalon de dessus : «La Dame épouse vous demande de vous y mettre sur-le-champ. Le reste viendra aussitôt après", dit-elle. Akoki répondit pour sa maîtresse qui était restée couchée derrière l'écran: "Je ne sais ce que c'est, mais Mademoiselle se sent indisposée depuis hier soir et se repose encore. Je lui en parlerai à son réveil." La servante s'en retourna. La Demoiselle voulut se lever pour se mettre à l'ouvrage, mais le capitaine la retint.

La scène rapportée après l'indication temporelle semble le prolongement de celle qui la précède immédiatement : même moment de la journée, mêmes personnages, dans les mêmes attitudes. Cependant, il est difficile de ne pas supposer l'existence d'un laps de temps entre les premières visites, qui semblent bien se situer au passage de l'automne à l'hiver (du neuvième au dixième mois) et le festival de Kamo, qui a lieu à la fin du onzième. En outre, un certain nombre de jours doivent s'écouler entre le retour du pèlerinage, l'annonce de la désignation et le début des travaux de couture. On relève des incohérences de chronologie dans la suite du roman et on ne peut exclure non plus une inadvertance du romancier, d'autant plus que nous ignorons tout des circonstances dans lesquelles le texte a été composé. En tout cas, qu'il s'agisse d'une maladresse involontaire ou d'un procédé conscient, un tel mode d'enchaînement présente l'avantage d'assurer un glissement dans le temps sans détruire le sentiment de la continuité. Le décompte minutieux des journées à certains moments du récit ne relève pas d'un procédé mécanique comme dans une chronique, mais est étroitement lié à la succession des événements.

Comme l'ont noté les critiques ${ }^{13}$, le temps du Roman de la Chambre basse est un temps dramatique, que sous-tend l'expérience vécue par les personnages, les conflits qui les opposent, les projets qu'ils poursuivent. Les moments d'attente ou d'équilibre alternent avec des accélérations, souvent marquées par un sentiment d'urgence. On peut voir alors Akoki se démultiplier pour remplir en temps voulu tous les rôles qu'elle doit assumer. Les indications temporelles absolues ou relatives qui situent les événements les uns par rapport aux autres (« le lendemain »...) ou encore par rapport au calendrier officiel, le repérage par rapport au passage de saison, sont de même nature que ceux que l'on trouve dans les écrits autobiographiques comme le Kagerō no nikki (Mémoires d'une éphémère). Cependant, le temps du Roman de la Chambre basse est un temps intersubjectif: il ne se rattache à aucune subjectivité particulière, mais naît de l'interaction entre plusieurs temporalités distinctes, dont aucune ne peut être considérée comme la temporalité centrale. En cela la technique romanesque du Roman de la Chambre basse se distingue de celle de la littérature intime, mais aussi du temps psychologique qui prévaut dans le Roman du Genji.

\section{L'organisation de l'espace}

14 La dimension dramatique qui préside à la construction du temps caractérise aussi celle de l'espace. L'ensemble de l'action de la première partie se déroule dans le quartier aristocratique de Kyōto, dans les résidences des personnages principaux: celle du ministre de la Droite, qu'habite Michiyori, et celle du conseiller moyen, où se situe la chambre basse. D'autres lieux sont évoqués et servent d'arrière-plan à l'action: le 
palais où se rendent en service Michiyori et les gendres du conseiller moyen, la résidence du général de Katano, que fréquente une des servantes de la maison, la demeure de la tante d'Akoki d'où parviennent lettres et objets, le temple d'Ishiyama, en dehors de la ville, où la famille se rend pour un pèlerinage de plusieurs jours, le sanctuaire de Kamo. La mention de ces lieux fait deviner, en arrière-plan des événements, la présence de la Capitale et de la société aristocratique qu'elle abrite, tout en laissant sur le devant de la scène les deux pôles que sont les deux résidences du conseiller moyen et du père de Michiyori. La première partie et le début de la seconde jusqu'à l'enlèvement de la Demoiselle sont marqués par un constant mouvement de vaet-vient entre ces deux lieux, à la faveur duquel Michiyori se rapproche dans un premier temps de la chambre basse, s'y installe, avant d'enlever la Demoiselle pour l'installer dans sa propre demeure, située sur la Deuxième avenue. Dans les échanges entre les deux lieux, un rôle essentiel est dévolu aux personnages secondaires, Akoki et l'officier de la Maison du prince héritier (tachiwaki 帯刀), son mari, intermédiaires indispensables. Dans la première partie du roman, l'initiative leur appartient pour une grande part. C'est l'officier qui, sur les confidences d'Akoki, décide de parler d'elle à son maître, c'est lui encore qui porte les premières lettres et organise la première visite et qui, involontairement, précipite la marche des événements, lorsqu'il laisse tomber une lettre de la Demoiselle pour Michiyori. Mais déjà, à partir de la première visite, Akoki avait pris le relais, se chargeant, malgré la position délicate où se trouve sa maîtresse, des préparatifs du mariage, sollicitant sa tante afin d'obtenir les vêtements et les ustensiles indispensables. La force de caractère et l'ingéniosité du personnage, ainsi que le dévouement de son mari sont sans cesse soulignés et constituent un ressort important de l'intrigue. À côté de la chambre basse, qui est au centre des événements au début du roman, un autre lieu prend un relief particulier : l'appartement d'Akoki, où s'arrête l'officier lors de ses visites, où arrivent et d'où partent les lettres, où passe également Michiyori pour se décrotter après la route, et qui devient ainsi un véritable lieu de passage entre l'extérieur et l'intérieur de la résidence, assurant le maintien du lien entre les héros au moment les plus difficiles.

15 Ainsi, deux types de scènes romanesques ${ }^{14}$ structurent la narration dans la première partie du Roman de la Chambre basse. La première à la dimension de la ville de Kyōto ou du moins de ses quartiers aristocratiques est constituée par tous les lieux pris dans le réseau de communication qui unit les personnages, à l'instar de ce qui se passe dans un roman épistolaire, genre dont le Roman de la Chambre basse, comme d'ailleurs les autres monogatari connus possède de nombreux traits ${ }^{15}$. La narration s'organise alors souvent en de très longues phrases servant de cadre aux dialogues qui y sont tenus. Ces phrases peuvent enjamber plusieurs lieux et couvrent nécessairement un laps de temps assez important. Telle est par exemple la longue phrase qui s'étend de la page 8 à la page 10 de l'édition originale, dont voici les principales articulations :

L'officier étant allé trouver le capitaine [...] «Tiens porte-lui ceci», ajouta le capitaine et il tendit une lettre à l'officier, qui la prit en maugréant. "De la part de Monseigneur! ", dit-il en tendant la lettre à Akoki [...] Akoki prit la lettre et la porta à sa maîtresse [...] Mais la Demoiselle ne répondait rien. Akoki lut la lettre. [...] Comme la Demoiselle ne montrait aucun intérêt, [...] elle quitta la pièce.

16 Ainsi, au cours de ce qui, dans le texte original, est une seule phrase, le lecteur est mené de la résidence du conseiller moyen où vivent la Demoiselle et Akoki aux appartements du capitaine, puis de nouveau à la chambre d'Akoki, et enfin à celle de sa maîtresse, 
tandis que sont enchâssées trois conversations : celle du capitaine avec l'officier, de l'officier avec Akoki et d'Akoki avec sa maîtresse, ainsi que le texte d'une lettre.

À côté de cette grande scène, se rencontrent des scènes plus réduites, dont les limites correspondent à celles de différents espaces physiques. Il s'agit d'abord de la résidence du conseiller moyen avec ses différentes divisions : la chambre basse et son extension, la chambre d'Akoki; les appartements des différents membres de la famille et les passages qui les relient, le réduit où est enfermée la Demoiselle et ses abords. À cela, il faut ajouter les appartements de Michiyori dans la résidence du ministre, son père, ainsi que les rues de Kyōto menant d'une résidence à l'autre. Le resserrement de l'espace s'accompagne d'un ralentissement de la vitesse du récit; les phrases sont généralement brèves. Le passage d'un lieu à un autre est assuré, comme nous l'avons $\mathrm{vu}$, par le déplacement des personnages ou par la circulation des messages, mais aussi par le simple changement de point de vue, qui permet au narrateur de nous amener très librement d'un lieu à un autre, selon des procédés rappelant ceux du montage cinématographique. Le meilleur exemple, sans doute humoristique, est l'épisode de la première nuit passée ensemble par Michiyori et la Demoiselle. Celle-ci est racontée selon deux points de vue, l'un situé dans la chambre de la Demoiselle où se déroule la scène et l'autre dans celle des domestiques, où parviennent les bruits assourdis de la scène principale :

Le capitaine s'approcha de la Demoiselle et la prit dans ses bras [...] Akoki qui avait entendu remonter le volet voulut se lever [...] Sans relâcher la Demoiselle, le capitaine se dévêtit [...] La chambre d'Akoki étant mitoyenne de celle de la Demoiselle, elle entendit les sanglots étouffés de sa maîtresse [...] Le capitaine parlait à la Demoiselle [...] Quand le messager vint annoncer l'arrivée de la voiture, l'officier dit à Akoki : «Va les prévenir ${ }^{16}$ ! "

L'angoisse de l'héroïne et l'embarras du héros sont relayés et redoublés par ceux d'Akoki et de l'officier, tout comme le sont les sentiments amoureux du premier couple par ceux du second, l'auteur jouant avec malice sur le contraste des situations et des conditions sociales.

Dans cette organisation proprement dramatique de l'espace, champ de forces où se rencontrent et s'affrontent les acteurs du drame, la chambre basse devient un enjeu central. Lieu de confinement, à l'écart de l'habitation principale, elle devient aussi un pôle de résistance, un espace indépendant où s'organise une vie qui peu à peu échappe au contrôle de la maison principale. Le projet d'Akoki passe par la transformation de l'espace occupé par sa maîtresse. Aux parois creusées de fentes par où l'on peut être observé, aux cloisons dont la marâtre contrôle l'ouverture ${ }^{17}$, se substitue au milieu de la première partie l'écran (kichō 几帳) prêté à Akoki par sa tante, véritable accessoire de théâtre, qui transforme l'espace nu et sans défense de la chambre basse en un abri. Il sert à dissimuler Michiyori, est susceptible d'être déplacé, constitue en même temps un abri et un poste d'observation. Il dédouble l'espace de la chambre au moment où, après le mariage, la pièce d'Akoki a perdu une partie de son importance comme espace intermédiaire. Un jeu analogue se met en place un peu plus loin, à la fin de la première partie, autour de la porte verrouillée qui interdit l'accès du réduit où se trouve enfermée l'héroïne et dont seule la marâtre contrôle en maître absolu l'ouverture et la fermeture. La perte par la Demoiselle de cette base, aussi fragile qu'elle ait été, marque sa défaite absolue. Mais la nouvelle porte qui déjoue la sagacité d'Akoki et limite très fortement les échanges avec la Demoiselle, se révèle dans la suite du récit comme la protection la plus sûre, à l'abri de laquelle la Demoiselle pourra attendre sa délivrance. Dès le début, la dépendance de l'héroïne s'exprime tout autant que par le confinement 
dans la chambre basse, par l'incapacité où elle est d'échapper aux regards. Le conseiller moyen, la marâtre sont au début en position d'observateurs, aux regards scrutateurs desquels rien ne saurait échapper. Mais l'introduction de l'écran transforme la structure de l'espace : d'observatrice, la marâtre devient à son tour un spectacle offert aux yeux ironiques de Michiyori.

Le roman tel que le conçoit l'auteur du Roman de la Chambre basse relève donc avant tout d'un art du point de vue. Lieu des regards croisés, il rend possible l'effacement derrière les personnages $\mathrm{du}$ narrateur, dont les jugements de valeur n'apparaissent qu'épisodiquement. Quant aux personnages, ils sont eux-mêmes constamment présentés à travers le regard des autres personnages. La Demoiselle est vue par les yeux compatissants de sa suivante Akoki, hostiles de la marâtre, émus, puis amoureux et admiratifs de Michiyori. L'image de la marâtre nous est donnée à travers le regard d'Akoki, d'une dame de compagnie, de Michiyori ou de la Demoiselle... Le romancier use volontiers de ce genre de notations, qui confèrent à la narration un caractère très concret et permettent d'inscrire dans la durée vivante du roman le commentaire descriptif ou psychologique. Ainsi cette notation visuelle, par laquelle se concluent les propos jaloux que tient Michiyori à la Demoiselle et qui nous rassure sur les sentiments du capitaine :

Ses mains occupées à coudre étaient d'une blancheur éclatante et d'une parfaite distinction ${ }^{18}$.

21 Et encore ces deux autres notations, qui nous font deviner respectivement les sentiments éprouvés par l'officier à la vue d'Akoki et par Akoki en voyant emmener loin d'elle sa maîtresse : toutes les deux ont comme la précédente cette particularité qu'elles s'attachent à saisir un objet en mouvement, à la fois dans le temps et dans l'espace :

Akoki, pimpante et parée, la ceinture lâchement nouée autour de ses hanches, et la chevelure, de près de trois shaku plus longue que sa taille, retombant dans le dos, s'éloigna, sous le regard admiratif de l'officier ${ }^{19}$.

Ou encore, plus loin :

La Dame épouse, empoigna la Demoiselle par la manche comme on se saisit d'un fuyard et la poussa devant elle, renversant les ustensiles sur son passage. La Demoiselle était vêtue d'une robe de soie mauve d'une parfaite souplesse par-dessus la tunique blanche également en soie que lui avait laissée le capitaine. Sa chevelure, dont elle avait commencé à prendre soin ces derniers temps, offrait un spectacle adorable. Elle ondulait dans son dos, dépassant sa taille de cinq bons pouces, et conférait à sa silhouette une élégance merveilleuse. Tandis qu'elle la regardait partir, se demandant avec angoisse ce que s'apprêtait à lui faire la Dame épouse, Akoki se sentit prise de vertige ${ }^{20}$.

L'art consommé avec lequel le romancier met en scène l'espace et le temps est démontré dans la scène du kaimami 坦間見 (littéralement : vision à travers une fente), au cours de laquelle Michiyori, comme il se doit pour un héros de roman, aperçoit pour la première fois la Demoiselle.

L'officier conduisit le capitaine à un endroit où la cloison était légèrement entrouverte et resta lui-même posté un moment sur la véranda, pour le cas où surviendraient des gardes. Le capitaine, en jetant le regard à l'intérieur, vit que la chambre était éclairée par une lampe prête à s'éteindre. Nul rideau, nul paravent n'obstruait la vue. Celle qui lui faisait face devait être Akoki : elle était bien faite, avec une tête délicatement dessinée, et portait une tunique blanche avec par-dessus une élégante robe (akome 衵) de soie lustrée. Celle qui reposait auprès d'elle était sans doute la Demoiselle. Elle était vêtue d'une tunique blanche manifestement usée, le bas de son corps couvert par ce qui devait être un manteau de soie lustrée, doublé d'ouate. Elle détournait le visage, si bien qu'il ne pouvait en apercevoir les 
traits. Il n'eut le temps que de remarquer le dessin délicat de sa tête (kashiratsuki) et la beauté de la chevelure qui lui retombait dans le dos, lorsque la lumière s'éteignit. Quoique dépité, le capitaine était bien décidé à ne pas en rester là. "Comme il fait noir! dit alors la Demoiselle. Mais tu m'as dit que tu avais de la visite. Va vite. » Même sa voix était de la plus grande élégance ${ }^{21}$.

Il faudrait, pour analyser en détail cette scène, la comparer à d'autres scènes de kaimami des autres romans de l'époque ${ }^{22}$. On voit cependant dès l'abord que le dispositif permet de créer une tension dramatique en laissant planer la menace d'une interruption venue de l'extérieur. Si aucun obstacle ne vient obstruer la vue du spectateur et si la Demoiselle apparaît dans la vulnérabilité de celle qui est exposée aux regards extérieurs, le cloisonnage très précis de l'espace et du temps empêche cependant Michiyori de satisfaire pleinement sa curiosité : il ne peut bénéficier que d'une vue très partielle et très brève. La "lampe prête à s'éteindre ", outre qu'elle révèle la condition misérable de l'héroïne, en contraste avec sa beauté et sa distinction, a aussi pour fonction de circonscrire la perception, de la découper, et de juxtaposer par succession dans le temps deux impressions de nature différente : visuelle et auditive, avec juste l'interstice nécessaire à la perception distincte de l'une et de l'autre. Fragmentaire et évanescente, l'image laisse toute sa place au désir d'en percevoir plus. En même temps, elle ne permet aucun doute: la convergence parfaite de ces deux perceptions distinctes est pour Michiyori la preuve irréfutable qu'il a trouvé ce qu'il cherchait.

Témoin de la situation du roman avant le Roman du Genji, le Roman de la Chambre basse, montre un niveau très élevé de perfection technique et de prise de conscience des procédés et possibilités romanesques. Utilisant pleinement les ressources de la langue japonaise, il apporte des solutions souvent brillantes aux différents problèmes que pose l'écriture du roman et relève de ce fait beaucoup plus de la littérature savante que de la littérature populaire. Ces traits lui confèrent, aux yeux d'un lecteur d'aujourd'hui, une apparence étonnamment moderne. On peut souligner le caractère théâtral, presque cinématographique, de cet univers romanesque, avec notamment la présence d'une trame narrative ininterrompue, dans laquelle les événements découlent les uns des autres au sein d'une durée à la fois continue et intersubjective, somme des temporalités particulières des personnages. Peut-on imaginer un lien entre cette poétique du roman avec l'art du rouleau illustré dont on sait qu'il lui était associé ? En tout cas, il n'est pas douteux que le Roman de la Chambre basse offre un riche terrain d'exploration tant par sa qualité propre que par ce qu'il nous apprend sur la poétique du roman de l'époque de Heian et en particulier du Roman du Genji qu'il permet de mieux situer dans l'histoire du genre romanesque.

\section{BIBLIOGRAPHIE}

Texte 
Ochikubo monogatari, gendaigotsuki-shinpan, volumes 1 et 2, Muroki Hideyuki (éd.), Kadokawa

shoten, 2004.

Ochikubo monogatari, Sumiyoshi monogatari, Fujii Sadakazu, Inaga keiji (éd.), Shin Nihon koten bungaku taikei 18, Iwanami shoten, 1989 (abréviation dans le texte : snkbt).

\section{Traduction}

Ochikubo monogatari or The Tale of Lady Ochikubo. A Tenth Century Japanese Novel, Whitehouse Wilfrid et Yanagisawa Eizō (trad.), The Hokuseidō Press, Tōkyō, 1965.

Études

Hijikata Yōichi, « Ijime no kōzō, Ochikubo monogatari ron », Aoyama gobun, n 20, 1990.

Imai Takuji, Monogatari bungaku shi no kenkȳu, Waseda daigaku shuppan bu, 1977.

Mauclaire Simone, Du conte au roman : un Cendrillon japonais du Xe siècle :l'Ochikubo monogatari, Maisonneuve et Larose, 1984.

Mitani Kuniaki, « Ochikubo no hōhō, sono kyōju to hyōgen o megutte », dans Heianchō bungaku kenkyū, II-8, février 1969. Repris dans Nihon bungaku kenkyū shiryō sōsho, Heian monogatari iii, Yūseidō, 1979.

Naganuma Eiji, Ochikubo monogatari no hyōgen kōsei, Shintensha, 1994.

\section{NOTES}

1. Les Notes de chevet de Sei Shōnagon, le Roman du Genji ou les Trois trésors illustrés (Sanbōe) de Minamoto no Tamenori (984), notamment, donnent des titres de romans dont le texte n'est pas parvenu jusqu'à nous. Ces monogatari qui allaient du roman d'amour au conte merveilleux étaient déjà très nombreux au $\mathrm{x}^{\mathrm{e}}$ siècle. La préface aux Trois trésors illustrés affirme qu'ils sont "plus nombreux que les herbes dans une forêt ou que le sable au bord de la mer ».

2. Long roman de cour en vingt volumes, anonyme, rédigé dans les années 970-990. Le Roman de la Caverne raconte des événements relatifs à une succession impériale en liaison avec le destin d'une famille de courtisans dépositaires de traditions musicales venues de la Chine.

3. Roman en quatre parties, anonyme, rédigé probablement autour de 990. Cf. la notice de l'édition de SNKBT. Le Roman de la Chambre basse est mentionné dans les Notes de chevet de Sei Shōnagon et semble avoir été à peu près oublié par la suite. Malgré les nombreuses spéculations sur l'auteur possible - on a pu proposer notamment le nom du poète Minamoto no Shitagō 源順 (911-983) et beaucoup de critiques considèrent qu'il s'agit sans doute d'un homme - il n'y a aucune certitude à ce sujet.

4. Voir l'étude de Mitani Kuniaki, «Ochikubo no hōhō, sono kyōju to hyōgen o megutte » (La méthode du Roman de la Chambre basse, à propos de sa réception et de son expression), dans Heianchō bungaku kenkyū, II-8, février 1969. Repris dans Nihon bungaku kenkyū shiryō sōsho, Heian monogatari III, Yūseidō, 1979.

5. L'étude du Roman de la Chambre basse a été d'abord entreprise par des savants du mouvement des Études Nationales (kokugaku) autour de Kamo no Mabuchi. Ces travaux sont à l'origine de l'édition de 1794, en caractères mobiles. Le texte a attiré également l'attention d'Ueda Akinari, auteur d'une autre édition en 1799, qui est assortie d'une préface où le romancier fait l'éloge des deux héros du roman et surtout de l'héroïne. Le texte d'Akinari, qui a procédé à des amendements et ajouté des indications marginales, est devenu la version commune et a servi de base aux éditions ultérieures jusque dans l'époque contemporaine. 
6. Un vibrant éloge des qualités littéraires du roman, notamment de son art de la construction et de son humour, est fait par ses traducteurs anglais Wilfrid Whitehouse et Yanagisawa Eizō dans la préface à leur traduction Ochikubo monogatari or The Tale of Lady Ochikubo, A Tenth Century Japanese Novel, The Hokuseidō Press, Tōkyō, 1965.

7. Dans le chapitre «Les Lucioles » du Roman du Genji, on trouve une indication sur le grand nombre de romans mettant en scène la méchanceté des marâtres.

8. Pour une lecture politique du Roman de la Chambre basse, voir l'étude de Hijikata Yōichi, « Ijime no kōzō, Ochikubo monogatari ron " («La structure de la persécution, étude sur le Roman de la Chambre basse »), Aoyama gobun, $\mathrm{n}^{\circ} 20,1990$. Hijikata souligne notamment le rôle important joué par la famille ou la maison (ie) et par les liens matrimoniaux dans la société du $\mathrm{x}^{\mathrm{e}}$ siècle. Des tensions opposaient des familles rivales ou les membres d'une même famille. «Le sujet de ce roman, écrit Hijikata, n'est rien d'autre que les luttes de pouvoir au sein du système fondé sur l'institution impériale: il s'agit d'un roman politique (seiji no monogatari), d'un roman sur le pouvoir (kenryoku no monogatari) vu à travers le prisme de la "Maison". »

9. Voir dans ce numéro l'article de Charlotte Von Verschuer http://cipango.revues.org/1029.

10. Le miscanthe en fleur (hanasusuki, obana) est associé au milieu de l'automne. En outre, l'expression ho ni idzu (former un épi) qu'appelle ce thème signifie aussi « se manifester » et donc « exprimer ses sentiments».

11. Le capitaine de Katano est le célèbre héros d'un roman perdu, mentionné également dans les Notes de Chevet et le Roman du Genji. Comme dans le Roman de la Chambre basse, dans le Roman du Genji le capitaine de Katano fait figure de parangon de galanterie (irogonomi) et c'est par référence à ce modèle et en contraste avec lui qu'est conçu le personnage du Genji, non moins volage, mais plus discret et aux sentiments moins superficiels. Selon une remarque du narrateur au début du deuxième chapitre, le capitaine de Katano se serait moqué du Genji s'il avait eu connaissance de ses aventures.

12. Le capitaine s'en va à l'aube, la Demoiselle achève son travail de couture et le remet à la marâtre. Puis vient une lettre du capitaine. En réponse, la Demoiselle lui renvoie la flûte qu'il a oubliée et reçoit de lui une nouvelle réponse. Cependant le paragraphe suivant nous apprend que dès le départ du capitaine, la marâtre va voir son mari, obtient son blanc-seing et vient chasser la Demoiselle de la chambre. L'efficacité dramatique et l'effet de précipitation du cours des événements ainsi obtenus le sont au prix d'un flou dans la chronologie des événements.

13. Cf., par exemple, Naganuma Eiji, Ochikubo monogatari no hyōgen kōsei, Shintensha, 1994, chapitre 2.

14. Nous appellerons ici scène romanesque toute séquence narrative caractérisée par une durée continue et par son inscription dans un lieu déterminé. Cela inclut des séquences qui peuvent se dérouler en même temps ou successivement dans plusieurs pièces distinctes d'une résidence ou même dans plusieurs points éloignés de la capitale, mais reliés par le déplacement des personnages ou le va-et-vient des messagers de sorte que la continuité du temps et du lieu soit respectée.

15. Sur le roman épistolaire de l'époque de Heian, voir l'étude d'Aileen Gatten, "Fact, Fiction, and Heian Literary Prose: Epistolary Narration in Tōnomine Shōshō Monogatari, Monumenta Nipponica, $\mathrm{n}^{\circ} 53,1998$.

16. SNKBT, pp. 25-27.

17. On le voit à la colère de la marâtre quand elle se heurte à une cloison fermée préalablement par Akoki, qui donne lieu à une scène de caractère très théâtral. "Pourquoi mettez-vous si longtemps à ouvrir? ", insiste la marâtre, tandis qu'Akoki se précipite pour rattraper le temps perdu (op. cit., p. 55).

18. Ibid., p. 78.

19. Ibid., p. 38. Le shaku est une mesure de longueur équivalente à 33 centimètres.

20. Ibid., p. 85 . 
21. Ibid., p. 23.

22. Le kaimami, scène au cours de laquelle un homme aperçoit une femme par un interstice dans la cloison, semble avoir été un poncif du roman de l'époque de Heian, où il joue un rôle analogue à la fameuse "scène de la première rencontre » étudiée par Jean Rousset dans Leurs Yeux se rencontrèrent, Corti, 1981. On en trouve à la même époque un exemple dans le Roman de la Caverne, et plusieurs dans le Roman du Genji : la scène où le Genji contemple Utsusemi (chap. Utsusemi [La mue de la cigale]), celle où il aperçoit pour la première fois Murasaki no ue (chap. Wakamurasaki [Jeune grémil]), celle où son frère peut voir Tamakazura à la lumière de lucioles lâchées par le Genji (chap. Hotaru [Les lucioles]), celle où Yūgiri, fils du Genji, observe Murasaki no ue, l'épouse de son père (chap. Nowaki [La tempête]), celle où Kashiwagi, demi-frère de Tamakazura, aperçoit par accident la Princesse troisième, épouse du Genji (chap. Wakana jōo [Jeunes herbes 1]), celle où le Lieutenant attaché à la Chancellerie, fils de Yūgiri, voit les filles de Tamakazura (chap. Takekawa [La rivière aux bambous]), celle enfin où Kaoru, que chacun croit être le fils du Genji, mais qui est en fait le fils de Kashiwagi, contemple les filles du Prince huitième, frère cadet du Genji (chap. Hashihime [Les jouvencelles du pont]).

\section{RÉSUMÉS}

Le Genji monogatari a éclipsé des œuvres antérieures ou contemporaines, parfois remarquables, dont l'étude permet d'en mieux saisir l'originalité. Ainsi le Roman de la chambre basse, dont est ici étudiée l'organisation spatiale et temporelle.

The Genji monogatari overshadowed several older works,some of which were notable in their own right. The study of these works can help to understand the originality of the Genji. This article studies the spatial and temporal structure of The Tale of Ochikubo.

\section{INDEX}

Thèmes : littérature

キーワード : Genji monogatari 源氏物語, monogatari 物語, Murasaki Shikibu 紫式部 (v. 973-v. 1014 ou 1025), Ochikubo monagatari 落坾物語, takonsei 多婚性, shōsetsu 小説, Heian jidai 平安 時代 (794-1185), bungaku 文学

Mots-clés : Dit du Genji, écriture, Genji monogatari, merveilleux (littérature), Murasaki Shikibu (v. 973-v. 1014 ou 1025), Ochikubo monagatari, polygamie, roman, Roman de la Chambre basse Keywords : Genji monogatari, Heian, Literature, Marvelous in Literature, monogatari, Ochikubo monagatari, Polygamy, Tale of Genji

Index chronologique : Heian 